\title{
El autismo en el primer año
}

\author{
Isabel Paula-Pérez, Josep Artigas-Pallarés
}

Resumen. Actualmente es posible diagnosticar el autismo con un alto grado de fiabilidad entre los 18 meses y los 2 años. Sin embargo, los primeros síntomas ya están presentes mucho antes del diagnóstico. Ello ha dado pie a que se hayan llevado a cabo múltiples estudios, retrospectivos y prospectivos, orientados a detectar manifestaciones que faciliten un diagnóstico lo más precozmente posible. A partir de estas investigaciones se han podido detectar síntomas cuya aparición se sitúa entre los 6 y 12 meses. Aunque se ha visto que estos síntomas tienen un interés diagnóstico limitado, aportan información muy valiosa para la comprensión del autismo en el marco de los trastornos del neurodesarrollo, en el sentido de destacar un patrón evolutivo que en su inicio es común a diversos trastornos, pero que progresivamente va configurando un fenotipo específico.

Palabras clave. Autismo. Detección temprana. Diagnóstico precoz. Estudios prospectivos. Estudios retrospectivos. Signos precoces. TEA. Trastorno del espectro autista. Trastornos del neurodesarrollo.

\section{Introducción}

El diagnóstico de autismo se suele confirmar entre los 3 y 6 años [1-3], si bien estas cifras varían en función de la zona geográfica (medio rural o urbano) y del entorno sociocultural. Sin embargo, es común que la preocupación familiar, o la sospecha de un problema del desarrollo, haya aparecido mucho antes. El intervalo de edad a partir del cual los padres recuerdan haberse preocupado seriamente por el desarrollo de su hijo se sitúa en los 15-18 meses [4]. Existe, por lo tanto, un retraso en el diagnóstico que genera problemas importantes, entre los cuales se suele citar la demora de la intervención terapéutica. De todas formas, sin restar importancia a este aspecto, las consecuencias van mucho más lejos. Por un lado, no se debe ignorar el sufrimiento de las familias ante la percepción evidente de un problema para el cual no se les ofrece una explicación cierta, y ante el que se minimiza su importancia o, simplemente, se niega su existencia. Por otra parte, sin diagnóstico no se pueden tomar en consideración medidas para prevenir el riesgo genético, muy elevado en los autismos sindrómicos asociados a discapacidad intelectual y no menospreciable en el autismo idiopático.

Los esfuerzos destinados a disminuir la edad de diagnóstico han promovido el diseño de diversas pruebas de cribado (p. ej., M-CHAT) y tests de confirmación diagnóstica (p. ej., ADI-R y ADOS) que permiten obtener un diagnóstico fiable para la mayor parte de pacientes con trastorno del espectro autista (TEA) alrededor de los 18 meses. No obs- tante, a sabiendas de que las manifestaciones del autismo se inician antes de esa edad, se están llevando a cabo múltiples estudios orientados a obtener un diagnóstico a edades más tempranas. Bajo esta perspectiva se han llegado a identificar diversos marcadores conductuales que sugieren que, por lo menos en una parte importante de pacientes con TEA, es posible obtener un diagnóstico fiable alrededor de los 14 meses. Los signos detectados a partir del primer año, replicados en varios estudios, se pueden agrupar en síntomas referidos a la comunicación social, a las conductas repetitivas -incluyendo estereotipias y uso atípico de objetos- y a las alteraciones en la regulación emocional [5].

No obstante, se han descrito síntomas incluso más precoces, pero cuya validez diagnóstica no se ha confirmado. Algunos de estos síntomas se refieren, entre otros, a aspectos muy precoces del desarrollo, como problemas del sueño [6], del llanto [7] o de la alimentación [8].

Esta revisión pretende recoger los datos más relevantes sobre las manifestaciones precoces del autismo, contempladas como una ayuda al diagnóstico precoz y como una aportación a la comprensión de la naturaleza del autismo.

\section{Métodos de investigación para la detección temprana del autismo}

La investigación relativa a la detección temprana del autismo ha pasado en los últimos años de los
Departamento de Métodos de Investigación y Diagnóstico en Educación, MIDE; Universitat de Barcelona; Barcelona (I. PaulaPérez). Unidad de Neuropediatría; Hospital de Sabadell; Centre Mèdic Psyncron; Sabadell, Barcelona, España (J. Artigas-Pallarés).

Correspondencia:

Dra. Isabel Paula Pérez. Campus Mundet. Departamento MIDE. Universitat de Barcelona. Edif. Llevant, 2. piso, despacho 264. E-08035 Barcelona.

E-mail:

isabelpaula@ub.edu.

Declaración de intereses: Los autores manifiestan la inexistencia de conflictos de interés en relación con este artículo.

Aceptado tras revisión externa: 10.01.14

Cómo citar este artículo: Paula-Pérez I, Artigas-Pallarés J. El autismo en el primer año. Rev Neurol 2014; 58 (Supl 1): S117-21. 
diseños retrospectivos, basados en información relatada por los padres y codificación de vídeos domésticos, a los estudios prospectivos longitudinales con bebés de riesgo de padecer autismo, especialmente aquellos cuyos hermanos mayores han recibido este diagnóstico.

Hasta fechas relativamente recientes, la mayor parte de las investigaciones han sido retrospectivas. Estos estudios no pueden obviar las limitaciones derivadas de la baja fiabilidad de la información aportada por los padres o del sesgo en la selección de comportamientos registrados, elegidos por motivos ajenos a la investigación [9-11]. Por el contrario, la investigación prospectiva examina la cronología y el curso de aparición de los síntomas conductuales del autismo en muestras de bebés de riesgo seguidos por equipos de investigación entrenados en el registro estandarizado de hitos considerados relevantes para el desarrollo normal o sospechosos de estar vinculados al autismo. Esos bebés de riesgo, generalmente hermanos de personas con autismo, se estima que tienen una probabilidad de padecer autismo alrededor de un $20 \%$ superior a la de la población general [12].

Se han utilizado dos métodos de codificación: la frecuencia de comportamientos sociocomunicativos específicos, observados durante la sesión, y la recogida, mediante la información de los padres o cuidadores, de datos sobre el comportamiento social. Con posterioridad se comparan estas variables entre los niños que reciban un diagnóstico definitivo de autismo y los que evolucionen neurotípicamente.

\section{Autismo de 'inicio temprano' o de "curso regresivo"}

El análisis de la información retrospectiva de los padres, de los vídeos domésticos y de los datos evolutivos prospectivos de niños en situación de riesgo ha permitido observar dos patrones: el autismo de 'inicio temprano', en el que los síntomas están presentes desde la primera infancia, y el autismo de 'curso regresivo', en el cual, después de un período de uno o dos años de desarrollo aparentemente normal, se observan síntomas relacionados con la pérdida de habilidades sociocomunicativas [9,13-16]. La regresión frecuentemente se acompaña de una reducción del interés y la motivación social [17]. Esta regresión suele ocurrir entre los 16-20 meses $[16,18,19]$.

La prevalencia de la regresión varía según diferentes estudios y depende del método de muestreo y de la definición de regresión. Algunos estudios detectan un curso regresivo en el 20-47\% de los niños, por lo general alrededor del segundo año de vida y a una edad media de 19-21 meses [16,20]. Otros estudios, basados en grandes muestras, informan de regresiones del 15,6\% [19] al 27\% [21].

Werner y Dawson [9] hallaron que los niños con patrón de inicio regresivo mostraban conductas de atención conjunta similares a la de niños de desarrollo típico al año de edad, pero a los 2 años manifestaban alteraciones de la comunicación social similares a las de los niños con un patrón de inicio temprano sin regresión. Además, mostraron un empeoramiento de la mirada social que se iniciaba a la finalización del primer año de vida.

Sí parece evidente que la asociación entre regresión social y otras variables (cociente de inteligencia, conducta adaptativa, gravedad de los síntomas o condiciones médicas) es muy variable $[15,22]$.

En un estudio más pormenorizado, Ozonoff et al [23] identificaron, a partir de la codificación del comportamiento social en vídeos domésticos y del registro prospectivo de hitos del desarrollo entre las edades de 6 y 24 meses, tres tipos diferentes de trayectorias: uno de inicio temprano; otro, de curso regresivo tras un periodo aparentemente normal, y un tercer tipo, de estancamiento sin regresión evidente. Se constató que la información aportada por los padres sobre el inicio temprano, la regresión o el estancamiento, determinantes para la asignación a uno de los tres grupos, no coincidía con la asignación basada en la observación prospectiva. Por otra parte, en el grupo de trayectoria regresiva se pudo poner en evidencia, en todos los casos, una disminución de la comunicación social antes de los 12 meses, en contraste con la regresión posterior y más tardía descrita por los padres. La principal conclusión del estudio fue que el autismo se inicia, de modo muy sutil y precoz, antes del año, inaparente para las familias, sin una marcada diferencia con la neurotipicidad, y que progresivamente, de forma más o menos aparente, se instaura una sintomatología que se ha tendido a interpretar como regresiva. Se pudo comprobar también que no existía una línea evolutiva divisoria entre los tres supuestos tipos: precoz, regresivo y de estancamiento.

En conjunto, los resultados de los estudios observacionales longitudinales sugieren que la regresión -es decir, la clara pérdida de habilidades- [23] puede ser el extremo de una serie de trayectorias que se caracterizan por un desarrollo lento o estancamiento en el lenguaje y habilidades cognitivas no verbales, además de las variables relacionadas con la comunicación social [23-26]. 


\section{Aportaciones fisiopatológicas}

El sustrato neuroestructural del autismo se atribuye a un mal funcionamiento del córtex asociativo motivado por desajustes en la conectividad intrahemisférica, caracterizados por la concomitancia de zonas de hipoconectividad y zonas de hiperconectividad, de donde se deriva una baja eficiencia en la integración de la información y en la coordinación entre los distintos sistemas neurales [27-29]. Bajo esta premisa se han llevado a cabo diversos estudios cuyo objetivo ha sido determinar la anisotropía fraccional (AF). La AF es un constructo que pertenece al campo de la física y que se refiere a la tendencia de cualquier elemento a seguir una trayectoria determinada, en oposición a la isotropía, que implica una ausencia de determinismo direccional (p. ej., la mayor o menor influencia de un campo magnético o la magnitud del efecto de una lente en la refracción de la luz). Aplicado al neurodesarrollo, la AF cuantifica la dependencia direccional de las trayectorias de las fibras nerviosas. Algunos estudios habían hallado en muestras de pacientes con TEA una baja AF [30], y otros trabajos, una elevada AF [31]. Una posible explicación a estos datos, aparentemente contradictorios, podría ser una discordancia determinada por las edades de los pacientes estudiados, puesto que la $\mathrm{AF}$, al ser un parámetro involucrado en la plasticidad neuronal y el neurodesarrollo, puede depender del estadio evolutivo y ser específico para distintas estructuras o funciones neuronales.

Bajo estas premisas, Wolff et al [32] llevaron a cabo un estudio prospectivo con 92 bebés, hermanos de niños con TEA. Se valoraron las propiedades microestructurales de las vías nerviosas de la sustancia blanca relacionadas con el autismo mediante el estudio por resonancia magnética de difusión. Esta técnica permite calcular en cada punto la distribución de las direcciones de difusión de las moléculas de agua (valor que mide la AF). Los autores hallaron que, al alcanzar los 6 meses, los hermanos de niños con autismo que posteriormente desarrollarían el trastorno tenían una AF elevada, lo cual sugiere una baja plasticidad cerebral. Curiosamente, a los 24 meses, los mismos pacientes tenían una AF baja. De estos hallazgos se infiere que las alteraciones en la trayectoria de las vías nerviosas se pueden observar antes del inicio de la aparición de síntomas clínicos propios de autismo. En resumen, lo que se desprende del estudio de Wolff et al es que, a edades muy tempranas, en los pacientes con TEA se observan patrones atípicos de conectividad que difieren en función de los circuitos y del estadio de desarrollo; pero, dato muy importan-
Tabla I. Manifestaciones conductuales detectadas entre los 6-12 meses mediante vídeos domésticos.

Retraso en el seguimiento de la cara $[35,36]$

Retraso en el seguimiento con la mirada cuando otra persona señala con el dedo [37]

Falta de respuesta al nombre [38-40]

Reducción del contacto ocular [11]

Reducción de la sonrisa social [35,36,40,41]

Disminución de gestos comunicativos, señalar con el dedo declarativamente [39,41,42]

Menor frecuencia en dirigir la mirada

y vocalizaciones a otras personas $[36,41]$

Orientación social [23]

Estereotipias [38]

Sutiles retrasos en el desarrollo motor [43]

te, no son específicos para ninguna región cerebral o dominio conductual [33], sugiriendo que lo que caracterizaría el autismo en sus estadios precoces no es tanto una localización específica, o incluso una conducta específica, sino una trayectoria evolutiva.

\section{Manifestaciones clínicas durante el primer año}

Las conductas regulatorias (llanto, alimentación y sueño) son, en su conjunto, uno de los motivos más comunes de consulta pediátrica en los primeros meses. Con el fin de verificar si los problemas relacionados con conductas regulatorias podían tener relación con el autismo, se llevó a cabo un estudio donde se comparó la frecuencia de consulta por estos motivos en diversos centros de salud entre un grupo de 208 preescolares diagnosticados de TEA y un grupo control [34]. El estudio mostró que los niños con TEA habían sido atendidos con mucha mayor frecuencia que el grupo control. Si bien los autores mencionaban la inespecificidad de estas manifestaciones, destacaban que podían tomarse en consideración como signo precoz de autismo, sobre todo cuando existe un riesgo genético.

Zwaigenbaum et al [5] llevaron a cabo una revisión de los estudios más relevantes sobre manifestaciones precoces del autismo. Hallaron numerosos trabajos que ponían en evidencia la presencia de 
Tabla II. Manifestaciones conductuales detectadas entre los 6-12 meses mediante estudios prospectivos.

Disminución de la orientación social [44]

Menor sincronía en la interacción con los padres [45]

Menor actividad física durante la interacción social [46,47]

Menor fijación visual en las caras y menor

respuesta evocada por la mirada [47]

manifestaciones que ya estaban presentes alrededor de los 6 meses. Tales hallazgos provenían de estudios con videos domésticos [11,23,35-43] (Tabla I) y de estudios prospectivos [44-47] (Tabla II).

Si bien las primeras manifestaciones del autismo están presentes antes del primer año, no está claro en qué medida son signos propios y específicos del autismo o pueden estar vinculados a un trastorno asociado, por ejemplo, a la discapacidad intelectual, presente en el $70 \%$ de casos de TEA.

Otra cuestión es que, aunque las conductas reseñadas en las tablas I y II se presentan con mayor frecuencia en niños con TEA que en neurotípicos, se desconoce la proporción de niños sin patología alguna que también han presentado estas conductas.

También es importante destacar que las conductas observadas en grupos de riesgo (hermanos menores de pacientes con TEA) son significativas cuando se compara el grupo de riesgo con un grupo control sin antecedentes de riesgo, pero cuando se intenta hallar diferencias significativas en estas conductas comparando los niños de riesgo que han desarrollado TEA con los que no lo han desarrollado, las diferencias apenas son significativas $[44,46,48]$.

\section{Conclusiones}

No cabe duda de que es posible un diagnóstico mucho más precoz del autismo de lo que se viene realizando actualmente, pero sigue latente la cuestión de a partir de qué momento se empieza a manifestar el autismo. De la revisión llevada a cabo se desprende:

- Los pacientes con autismo muestran síntomas a edades muy tempranas, alrededor de los 6 meses, o incluso antes.

- Los síntomas muy precoces, presentes antes del año, son poco específicos y no tienen por sí mismos valor diagnóstico.

- Las manifestaciones muy precoces del autismo sugieren una disfunción inespecífica del sistema nervioso, común a otros trastornos del neurodesarrollo que comparten aspectos genéticos y neurobiológicos.

- La especificidad del TEA estaría vinculada a influencias genéticas, tanto generalistas como específicas, y epigenéticas. Unas y otras, de forma interactiva, al margen de influir en un fenotipo común muy precoz, marcarían un patrón de neurodesarrollo, determinante de la evolución hacia el autismo, hacia otro trastorno o hacia la normalidad.

\section{Bibliografía}

1. Parner ET, Schendel DE, Thorsen P. Autism prevalence trends over time in Denmark: changes in prevalence and age at diagnosis. Arch Pediatr Adolesc Med 2008; 162: 1150-6.

2. Wiggins LD, Baio J, Rice C. Examination of the time between first evaluation and first autism spectrum diagnosis in a population-based sample. J Dev Behav Pediatr 2006; 27 (Suppl 2): S79-87.

3. Yirmiya N, Charman T. The prodrome of autism: early behavioral and biological signs, regression, peri- and post-natal development and genetics. J Child Psychol Psychiatry 2010; 51: 432-58.

4. Hernández JM, Artigas-Pallarés J, Martos-Pérez J, PalaciosAntón S, Fuentes-Biggi J, Belinchón-Carmona M, et al. Guía de buena práctica para la detección temprana de los trastornos del espectro autista. Rev Neurol 2005; 41: 237-45.

5. Zwaigenbaum L, Bryson S, Garon N. Early identification of autism spectrum disorders (I). Behav Brain Res 2013; 251: 133-46.

6. Richdale AL, Schreck KA. Sleep problems in autism spectrum disorders: prevalence, nature, and possible biopsychosocial aetiologies. Sleep Med Rev 2009; 13: 403-11.

7. Tongerloo M, Bor H, Lagro-Janssen A. Detecting autism spectrum disorders in the general practitioner's practice. Dev Disord 2012; 42: 1531-8.

8. Keen DV. Childhood autism, feeding problems and failure to thrive in early infancy. Seven case studies. Eur Child Adolesc Psychiatry 2008; 17: 209-16.

9. Werner E, Dawson G. Validation of the phenomenon of autistic regression using home videotapes. Arch Gen Psychiatry 2005; 62: 889-95.

10. Clifford S, Young R, Williamson P. Assessing the early characteristics of autistic disorder using video analysis J Autism Dev Disord 2007; 37: 301-13.

11. Clifford SM, Dissanayake C. The early development of joint attention in infants with autistic disorder using home video observations and parental interview. J Autism Dev Disord 2008; 38: 791-805.

12. Ozonoff S, Young GS, Carter A, Messinger D, Yirmiya N, Zwaigenbaum L, et al. Recurrence risk for autism spectrum disorders: a Baby Siblings Research Consortium study. Pediatrics 2011; 128: e488-95.

13. Ozonoff S, Heung K, Byrd R, Hansen R, Hertz-Picciotto I. The onset of autism: patterns of symptom emergence in the first years of life. Autism Res 2008; 1: 320-8.

14. Ozonoff S, Williams BJ, Landa R. Parental report of the early development of children with regressive autism: the delaysplus-regression phenotype. Autism 2005; 9: 461-86.

15. Richler J, Luyster R, Risi S, Hsu WL, Dawson G, Bernier R, et al. Is there a 'regressive phenotype' of autism spectrum disorder associated with the measles-mumps-rubella vaccine? A CPEA Study. J Autism Dev Disord 2006; 36: 299-316.

16. Goldberg WA, Osann K, Filipek PA, Laulhere T, Jarvis K, Modahl C, et al. Language and other regression: assessment and timing. J Autism Dev Disord 2003; 33: 607-16. 
17. Rogers SJ. Developmental regression in autism spectrum disorders. Ment Retard Dev Disabil Res Rev 2004; 10: 139-43.

18. Shinnar S, Rapin I, Arnold S, Tuchman RF, Shulman L, Ballaban-Gil K, et al. Language regression in childhood. Pediatr Neurol 2001; 24: 183-9.

19. Fombonne E, Chakrabarti S. No evidence for a new variant of measles-mumps-rubella-induced autism. Pediatrics 2001 108: E58.

20. Davidovitch M, Glick L, Holtzman G, Tirosh E, Safir MP. Developmental regression in autism: maternal perception. J Autism Dev Disord 2000; 30: 113-9.

21. Lingam R, Simmons A, Andrews N, Miller E, Stowe J, Taylor B. Prevalence of autism and parentally reported triggers in a north east London population. Arch Dis Child 2003; 88: 666-70.

22. Baird G, Charman T, Pickles A, Chandler S, Loucas T, Meldrum D, et al. Regression, developmental trajectory and associated problems in disorders in the autism spectrum: the SNAP study. J Autism Dev Disord 2008; 38: 1827-36.

23. Ozonoff S, Iosif AM, Young GS, Hepburn S, Thompson M, Colombi C, et al. Onset patterns in autism: correspondence between home video and parent report. J Am Acad Child Adolesc Psychiatry 2011; 50: 796-806.

24. Ozonoff S, Iosif AM, Baguio F, Cook IC, Hill MM, Hutman T, et al. A prospective study of the emergence of early behavioral signs of autism. Psychiatry 2010; 49: 256-66.

25. Landa RJ, Gross AL, Stuart EA, Bauman M. Latent class analysis of early developmental trajectory in baby siblings of children with autism. J Child Psychol Psychiatry 2012; 53: 986-96.

26. Landa R, Gross A, Stuart E, Faherty A. Developmental trajectories in children with and without autism spectrum disorders: the first 3 years. Child Dev 2012; 84: 429-42.

27. Dinstein I, Pierce K, Eyler L, Solso S, Malach R, Behrmann M, et al. Disrupted neural synchronization in toddlers with autism. Neuron 2011; 70: 1218-25.

28. Di Martino A, Kelly C, Grzadzinski R, Zuo XN, Mennes M, Mairena MA, et al. Aberrant striatal functional connectivity in children with autism. Biol Psychiatry 2011; 69: 847-56.

29. Minshew NJ, Williams DL. The new neurobiology of autism: cortex, connectivity, and neuronal organization. Arch Neurol 2007; 64: 945-50.

30. Shukla DK, Keehn B, Müller RA. Tract-specific analyses of diffusion tensor imaging show widespread white matter compromise in autism spectrum disorder. J Child Psychol Psychiatry 2011; 52: 286-95.

31. Weinstein M, Ben-Sira L, Levy $Y$, Zachor DA, Ben Itzhak E, Artzi M, et al. Abnormal white matter integrity in young children with autism. Hum Brain Mapp 2011; 32: 534-43.

32. Wolff JJ, Gu H, Gerig G, Elison JT, Styner M, Gouttard S, et al. Differences in white matter fiber tract development present from 6 to 24 months in infants with autism. Am J Psychiatry 2012; 169: 589-600.

33. Palau-Baduell M, Salvadó-Salvadó B, Clofent-Torrentó M,
Valls-Santasusana A. Autismo y conectividad neural. Rev Neurol 2012; 54 (Supl 1): S31-9.

34. Barnevik Olsson M, Carlsson LH, Westerlund J, Gillberg C, Fernell E. Autism before diagnosis: crying, feeding and sleeping problems in the first two years of life. Acta Paediatr 2013; 102: 635-9.

35. Adrien JL, Lenoir P, Martineau J, Perrot A, Hameury L, Larmande $\mathrm{C}$, et al. Blind ratings of early symptoms of autism based upon family home movies. J Am Acad Child Adolesc Psychiatry 1993; 32: 617-26.

36. Maestro S, Muratori F, Cesari A, Cavallaro MC, Paziente A, Pecini C, et al. Course of autism signs in the first year of life. Psychopathology 2005; 38: 26-31.

37. Maestro S, Muratori F, Cavallaro MC, Pei F, Stern D, Golse B, et al. Attentional skills during the first 6 months of age in autism spectrum disorder. J Am Acad Child Adolesc Psychiatry 2002; 41: 1239-45.

38. Baranek GT. Autism during infancy: a retrospective video analysis of sensory-motor and social behaviors at 9-12 months of age. J Autism Dev Disord 1999; 29: 213-24.

39. Osterling JA, Dawson G, Munson JA. Early recognition of 1-year-old infants with autism spectrum disorder versus mental retardation. Dev Psychopathol 2002; 14: 239-51.

40. Werner E, Dawson G, Osterling J, Dinno N. Brief report: recognition of autism spectrum disorder before one year of age: a retrospective study based on home videotapes. J Autism Dev Disord 2000; 30: 157-62.

41. Maestro S, Muratori F, Barbieri F, Casella C, Cattaneo V, Cavallaro MC, et al. Early behavioral development in autistic children: the first 2 years of life through home movies. Psychopathology 2001; 34: 147-52.

42. Osterling J, Dawson G. Early recognition of children with autism: a study of first birthday home videotapes. J Autism Dev Disord 1994; 24: 247-57.

43. Ozonoff S, Young GS, Goldring S, Greiss-Hess L, Herrera AM, Steele J, et al. Gross motor development, movement abnormalities, and early identification of autism. J Autism Dev Disord 2008; 38: 644-56.

44. Bhat AN, Galloway JC, Landa RJ. Social and non-social visual attention patterns and associative learning in infants at risk for autism. J Child Psychol Psychiatry 2010; 51: 989-97.

45. Yirmiya N, Gamliel I, Pilowsky T, Feldman R, Baron-Cohen S, Sigman M. The development of siblings of children with autism at 4 and 14 months: social engagement, communication, and cognition. J Child Psychol Psychiatry 2006; 47: 511-23.

46. Wan MW, Green J, Elsabbagh M, Johnson M, Charman T, Plummer F, et al. Parent-infant interaction in infant siblings at risk of autism. Res Dev Disabil 2012; 33: 924-32.

47. Key AP, Stone WL. Processing of novel and familiar faces in infants at average and high risk for autism. Dev Cogn Neurosci 2012; 2: 244-55.

48. Zwaigenbaum L, Bryson S, Rogers T, Roberts W, Brian J, Szatmari P. Behavioral manifestations of autism in the first year of life. Int J Dev Neurosci 2005; 23: 143-52.

\section{Autism in the first year}

Summary. At present autism can be diagnosed with a high degree of reliability between the ages of 18 months and 2 years. Yet, the first symptoms are already present long before the diagnosis is made. This has led to a number of retrospective and prospective studies being conducted with the aim of detecting manifestations that allow a diagnosis to be reached as early as possible. The results of these studies have enabled researchers to detect symptoms that appear between the ages of 6 and 12 months. Although it has been observed that these symptoms are of limited diagnostic interest, they do provide invaluable information for the understanding of autism within the framework of neurodevelopmental disorders because they highlight a pattern of development that is initially common to several different disorders, but which progressively goes on to constitute a specific phenotype.

Key words. ASD. Autism. Autistic spectrum disorder. Early detection. Early diagnosis. Early signs. Neurodevelopmental disorders. Prospective studies. Retrospective studies. 